\title{
GRBs as rare stages of stellar evolution
}

\author{
E. P. J. van den Heuvel \\ Sterrenkundig Instituut "Anton Pannekoek", University of Amsterdam, \\ The Netherlands \\ email: edvdh@science.uva.nl
}

\begin{abstract}
The association of "long" Gamma-Ray Bursts (durations $>2$ seconds) with peculiar Type Ic supernovae suggests strongly that this type of GRB is produced by the collapse of the rapidly rotating core of an initially very massive star to a black hole. At the time of collapse the star has lost its hydrogen-rich envelope and the GRB is thought to be produced by a collimated relativistic jet of matter ejected along the star's rotation axis. The angular momentum constraints for producing such a "collapsar" or "hypernova" suggest that the GRB-producing core collapses constitute only a small fraction of all core collapses of massive stars. As to the short-duration GRBs ( $<2$ seconds), which make up about one third of all GRBs, the most favoured model is that of the coalescence of a double neutron star or of a neutron star-black hole binary. Also these events are expected to be very rare, having a frequency of at most one event per hundred thousand years for a galaxy like our own. Due to the collimation of the relativistically ejected matter, the observable frequency of GRB events will, like in the case of the "long" bursts, be at least a factor hundred smaller.
\end{abstract}

\section{Discussion}

COURVOISIER: Is what we know of NS population and binary evolution compatible with the observed rate of short GRBs?

VAN DEN Heuvel: Berger et al. (2005), who discovered the short GRB 050724, argue in their paper that the observed incidence of short bursts, corrected for beaming (which makes that we see only 1 per cent of the GRBs produced by the mergers), is at least one order of magnitude lower than the expected merger rate of double NSs in galaxies. So, it appears that only a small fraction of the mergers succeeds in making a (short) GRB.

KRYVDYK: Very strong magnetic field is present in SN and BH. Is this fact taken into account in GRB models?

VAN DEN HEUVEL: Yes, this might be another parameter that could determine whether or not a NS + NS or NS + BH merger makes a Gamma Ray Burst.

MiRABEL: Do GRBs require merger of the two massive stars? Could BH binaries in our Galaxy be fossils of GRB Sources?

VAN DEN HEUVEL: Langer has suggested that only in the case of a merger of two helium cores one will have sufficient angular momentum (rotation) to produce a GRB when the core collapses to a black hole. This would be then a very rare event. I personally think that a helium star produced by mass transfer in a close binary could still be kept in sufficiently rapid rotation (by tidal forces) to produce a GRB. The helium-star (Wolf-Rayet) binary should then be very close (like V444 Cygni) for this to work. 